\title{
Towards Highly Improved Quark Actions *
}

\author{
M.G. Alford ${ }^{a}$, T.R. Klassen ${ }^{b}$, G.P. Lepage ${ }^{c}$

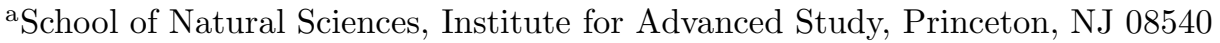 \\ bSCRI, Florida State University, Tallahassee, FL 32306 \\ cNewman Laboratory of Nuclear Studies, Cornell University, Ithaca, NY 14853, USA
}

We describe two ideas useful in the construction of highly improved quark actions for simulations on coarse lattices: (i) Field transformations solve the doubler problem without destroying tree-level improvement for on- or off-shell quantities. The simplest example is the Sheikholeslami-Wohlert (clover) action. Going to the next order of improvement yields the class of D234 actions. (ii) Anisotropic lattices with $a_{t}<a_{s}$ are useful because they push up the energy of unphysical branches of the dispersion relation (which are generic to highly improved actions), allow accurate mass determinations for particles with bad signal/noise properties (glueballs, P-state mesons), and enable one to simulate heavy quarks within a relativistic framework. We present first simulation results for the quenched light hadron and charmonium spectra obtained with a D234 action on anisotropic lattices.

\section{Introduction}

The last few years have seen a revival of the Symanzik improvement program [1-3]. The impetus was largely provided by tadpole improvement (TI) [4], which yields a simple first estimate of the large perturbative corrections to the treelevel coefficients in an improved action. In the case of non-relativistic QCD [5] and pure glue [6], the TI of tree-level coefficients has lead to accurate results on lattices as coarse as $a=0.4 \mathrm{fm}$.

Improved glue has classical $O\left(a^{4}\right)$ errors, and after TI any remaining quantum errors (of which the leading $\mathcal{O}\left(a^{2} \alpha\right)$ piece is known [2] and can be eliminated) seem to be small [6]. For quarks, the Sheikholeslami-Wohlert (SW) action [3], which has classical $\mathcal{O}\left(a^{2}\right)$ errors, is the most improved action that has been used in large-scale simulations. For full QCD simulations to be possible on coarse lattices, it therefore seems mandatory to design more highly improved quark actions $[7,8]$. Since exploratory simulations will have to be performed in the quenched approximation, for which the $a=0$ hadron spectrum is not a priori known,

\footnotetext{
*Based on talks by M.G.A. and T.R.K. Research conducted at the Cornell Theory Center and at SCRI, supported by DOE grants DE-FG05-85ER25000, DEFG05-92ER40742, DE-FG02-90ER40542 and the Monell Foundation.
}

it is important to have simple improvement tests for a quark action. As such we propose:

(i) The "effective velocity of light" $c(\mathbf{p})$ (defined by $\left.c(\mathbf{p})^{2}=\left(E(\mathbf{p})^{2}-E(0)^{2}\right) /|\mathbf{p}|^{2}\right)$ of various hadrons. Its deviation from 1 for non-zero masses and momenta is a measure of scaling violations.

(ii) The "r-test". For actions with Wilson and clover terms one can obtain a rough estimate of the correctness of the coefficient of the clover term, by seeing whether the spectrum is invariant under (small) changes of the Wilson parameter $r$. (iii) The scaling of dimensionless quantities such as $m_{N} / m_{\rho}, m_{\Delta} / m_{\rho}$, and $J$ [9].

(iv) The lattice spacing dependence of the rho mass in units of one of the standard scales (string tension, charmonium $\mathrm{S}-\mathrm{P}$ splitting, etc). This is a sensitive probe of scaling violations, but it requires that the scaling, systematic and measurement errors of the chosen scale be considerably smaller then those of the rho mass.

Last year we presented results for the "isotropic D234" quark action [7], which has classical $\mathcal{O}\left(a^{3}\right)$ errors. It gave very impressive results for all hadron mass ratios and the dispersion relation of mesons, much better than results from the SW action. However, the absolute value of the rho mass was almost identical to that of the SW action [10] for the three lattice spacings where they could be 
compared. To understand this somewhat incongruous behavior we have started to explore a variety of improved quark actions. Our aim is to disentangle the various sources of scaling errors.

One possible source of scaling violations is the unphysical branches in the quark dispersion relation that are generically present in actions improved beyond $\mathcal{O}(a)$. This has lead us to the study of actions on anisotropic lattices, and, in particular, to devise a simple procedure to construct actions that are tree-level improved to any order and doubler-free on an arbitrary lattice [11]. Working on anisotropic lattices with $a_{t}<a_{s}$ has several advantages:

(a) By decreasing $a_{t}$ one can decouple the unphysical branches by pushing up their energy.

(b) Larger and more easily identifiable effective mass plateaux lead to more accurate and confident mass determinations. This is important for particles with bad signal/noise properties, like Pstate mesons and especially [12] glueballs.

(c) Small $a_{t}$ allows one to simulate heavy quarks within a relativistic framework without the prohibitive cost of a fine spatial lattice.

The disadvantage of anisotropic lattices is that there are more independent coefficients in an improved action, and they have to be tuned to restore space-time exchange symmetry. One hopes that suitable TI gives reasonable estimates of (most of) the coefficients, also on anisotropic lattices, and our tests indicate that in general $([7,13]$, Sect. 3$)$ this appears to be the case. It is always better, of course, to tune them nonperturbatively. We briefly discuss this possibility in sect. 4. Tuning the clover term is important already for the isotropic case. It could solve the one problem of the isotropic D234 action, its low rho mass [7].

\section{Classically Improved Quark Actions}

There is a simple procedure to construct tree-level improved quark actions on an arbitrary lattice without a doubler problem. Let $a_{\mu}$ be the lattice spacings of a generic hypercubic lattice. $\left[a_{0} \equiv a_{t}\right.$; when the spatial lattice spacings $a_{i}$ are identical they will be denoted by $a_{s}$, such a lattice with $\xi \equiv a_{s} / a_{t}$ will be referred to as a ' $\xi: 1$ lattice'].
The idea is described in four steps:

(i) Start with a naive (improved) fermion action, $\bar{\psi}_{c} M_{c} \psi_{c}, M_{c}=\nabla_{c}+m_{c}$, where $\nabla_{c \mu}$ is an (improved) discretization of the continuum Dirac operator $D_{\mu}$, differing from it at $\mathcal{O}\left(a_{\mu}^{n}\right)$, say. The subscript 'c' stands for 'continuum-like', since this action is manifestly improved to $\mathcal{O}\left(a^{n}\right)$. However, this action will have doublers. The simplest choice of $\nabla_{c \mu}$, which leads to the SW action, is the standard anti-hermitean covariant derivative,

$\nabla_{\mu} \psi(x) \equiv \frac{1}{2 a_{\mu}}\left[\psi_{+\mu}-\psi_{-\mu}\right]=D_{\mu}+\mathcal{O}\left(a_{\mu}^{2}\right)$

with $\psi_{ \pm \mu} \equiv U_{ \pm \mu}(x) \psi(x \pm \mu)$. We will be interested in higher orders of improvement, as exemplified by the improved derivative

$\nabla_{c \mu}=\nabla_{\mu}-\frac{1}{6} a_{\mu}^{2} \nabla_{\mu} \Delta_{\mu}=D_{\mu}+\mathcal{O}\left(a_{\mu}^{4}\right)$.

Here $\Delta_{\mu}$ is the standard second order derivative,

$\Delta_{\mu} \psi(x) \equiv \frac{1}{a_{\mu}^{2}}\left[\psi_{+\mu}+\psi_{-\mu}-2 \psi(x)\right]$.

(ii) To cast the action in a form where doublers can easily be eliminated, perform a field transformation $\psi_{c}=\Omega \psi, \bar{\psi}_{c}=\bar{\psi} \bar{\Omega}$, so that $\bar{\psi}_{c} M_{c} \psi_{c}=$ $\bar{\psi} M_{\Omega} \psi$, where $M_{\Omega} \equiv \bar{\Omega} M_{c} \Omega$. We choose $\Omega=\bar{\Omega}$ (when acting to the right) and

$\bar{\Omega} \Omega=1-\frac{1}{2} r a_{0}\left(\nabla_{c}-m_{c}\right)+\mathcal{O}\left(a^{n}\right)$,

in terms of the (initially) free parameter $r$, the Wilson parameter. The transformed fermion operator $M_{\Omega}$ reads at this point

$M_{\Omega}=m_{c}\left(1+\frac{1}{2} r a_{0} m_{c}\right)+\not_{c}-\frac{1}{2} r a_{0} \nabla_{c}^{2}+\mathcal{O}\left(a^{n}\right)$.

This action still has doublers.

(iii) To remove the doublers, use

$\not_{c}^{2}=\Delta_{\mu}-\frac{1}{12} a_{\mu}^{2} \Delta_{\mu}^{2}+\frac{1}{2} \sigma \cdot F+\ldots$

to the appropriate order to eliminate $\nabla_{\mu}$ in terms of $\Delta_{\mu}$. Here $\sigma \cdot F \equiv \sum_{\mu \nu} \sigma_{\mu \nu} F_{\mu \nu}$ is the clover term, with $F_{\mu \nu}$ a lattice representation of the field strength, improved to the appropriate order. Since in momentum space $\Delta_{\mu}$ does not vanish at the edge of the Brillouin zone, the action obtained 
by the above truncation is doubler-free. This action, denoted by $\bar{\psi} M \psi$, is on-shell improved up to $\mathcal{O}\left(a^{n}\right)$ errors. If one is only interested in spectral quantities, one can stop here.

(iv) To (classically) also improve non-spectral quantities, undo the field transformation, which, e.g., amounts to using the propagator $G=$ $\Omega M^{-1} \bar{\Omega}[15]$. Note that this step does not reintroduce the doublers, since it was the truncation in step (iii), not the field transformation per se, that eliminated them.

We emphasize that in this approach one does not have to separately check the improvement of the interactions; it follows from the fact that they are improved for the naive action we started with, and this fact is not affected by the field transformation (the Jacobian of the latter can be ignored to the order we are working).

Let us explicitly write down the actions $M$ obtained for $n=2$ and 4: The SW action

$$
M_{\mathrm{SW}}=m_{0}+\not \nabla-\frac{1}{2} r a_{0}\left(\sum_{\mu} \Delta_{\mu}+\frac{1}{2} \sigma \cdot F\right),
$$

and the D234 action

$$
\begin{aligned}
M_{\mathrm{D} 234}= & m_{0}+\sum_{\mu} \gamma_{\mu} \nabla_{\mu}\left(1-b_{\mu} a_{\mu}^{2} \Delta_{\mu}\right) \\
& +c_{\mu} a_{\mu}^{3} \Delta_{\mu}^{2}-\frac{1}{2} r a_{0}\left(\sum_{\mu} \Delta_{\mu}+\frac{1}{2} \sigma \cdot F\right),
\end{aligned}
$$

where $m_{0} \equiv m_{c}\left(1+\frac{1}{2} r a_{0} m_{c}\right)$ in both cases, and

$b_{\mu}=1 / 6, \quad c_{\mu}=r a_{0} /\left(24 a_{\mu}\right)$.

We now choose $r$ to lead to as few and high lying unphysical branches in the free dispersion relation as possible. The general D234 action (8) has four branches $E=E(\mathbf{p})$ (not counting the particle anti-particle symmetry $E \leftrightarrow-E)$, of which three are unphysical. One easily shows:

- For $b_{0}=2 c_{0}$ there will be at most two unphysical branches.

- If furthermore $r=1-2 b_{0}$ or $b_{0}=0$ there is (at most) one unphysical branch.

- There are no unphysical branches if and only if $r=1, b_{0}=c_{0}=0$.

For the SW action $r=1$ is therefore the canonical choice. For a D234 action obtained as above one will have at least one unphysical branch; exactly one if one chooses $r=\frac{2}{3}$ and $c_{0}=\frac{1}{12}$, in addition to $b_{0}=\frac{1}{6}$. To obtain an isotropic D234

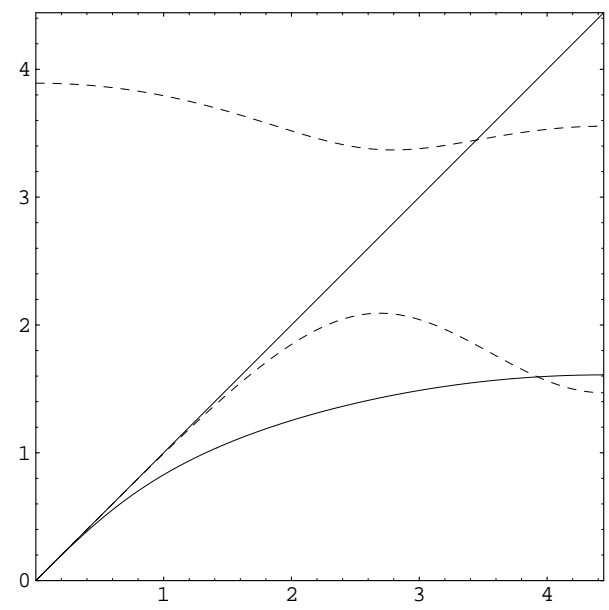

Figure 1. $a_{s} E(\mathbf{p})$ vs $a_{s}|\mathbf{p}|$, with $\mathbf{p} \propto(1,1,0)$, for massless SW action on a 1:1 lattice (solid), and D234 $\left(\frac{2}{3}\right)$ on a 2:1 lattice (dashed). Continuum fermions (thin solid) are shown for comparison.

action (on an isotropic lattice) one must choose all $b_{\mu}=\frac{1}{6}, c_{\mu}=\frac{1}{12}$ and $r=\frac{2}{3}$. Since the $c_{\mu}$ violate (9), this action [7] has $\mathcal{O}\left(a^{3}\right)$ errors. Like the $r=1 \mathrm{SW}$ action, it can be coded very efficiently using the "projection trick".

Requiring just one unphysical branch is not compatible with (9). Suitably modifying $c_{0}$ (and/or $b_{0}$ at $\left.\mathcal{O}\left(a_{0}\right)\right)$ to have just one branch leads to actions with $\mathcal{O}\left(a_{0}^{3}, a_{\mu}^{4}\right)$ errors. On anisotropic lattices with $a_{s} / a_{t} \geq 2$, say, one can presumably tolerate $\mathcal{O}\left(a_{0}^{3}\right)$ errors. In the free case it is even possible to avoid these $\mathcal{O}\left(a_{0}^{3}\right)$ errors; this is achieved [11] by a more complicated field transformation than (4). It leads to a D234 action where (some of) the coefficients have a slight mass dependence. Its spatial coefficients satisfy (9) and for the temporal ones, $r=1-2 b_{0}=1-4 c_{0}=$ $\frac{2}{3}\left(1+\frac{3}{4} a_{0} m_{c}\right) /\left(1+\frac{7}{12} a_{0} m_{c}\right)$. We will denote this action as D234 $\left(\frac{2}{3}\right)$, labelling it by the value of $r$ at $m_{c}=0$. This is the action used in our preliminary simulations. For details and some variations of the above procedure we refer to [11].

In figures 1 and 2 we compare the free quark dispersion relation of $\mathrm{D} 234\left(\frac{2}{3}\right)$ on a 2:1 with that of SW on a 1:1 lattice. Note particularly the (ex- 


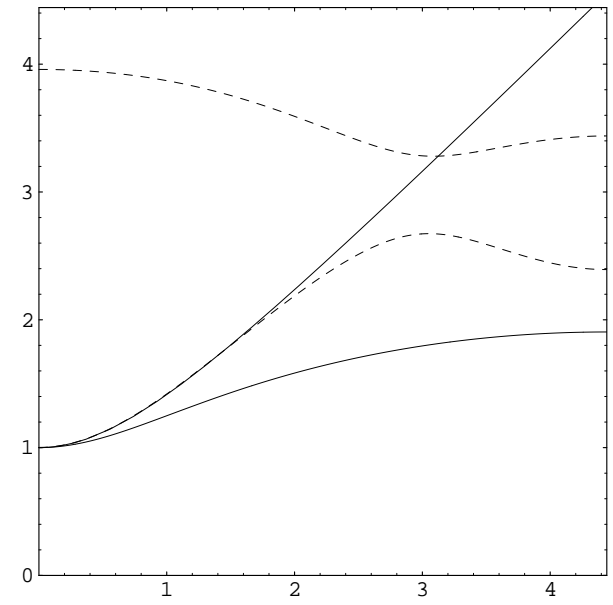

Figure 2. As in figure 1, for masses such that $a_{s} E(0)=1$ in all cases.

pected) dramatic improvement observed in the heavy mass case.

\section{Simulation Results}

The anisotropic Symanzik-improved gluon action as well as the TI prescription we used is described in [12]. In addition to $O\left(a_{s}^{4}\right)$ and $O\left(a_{s}^{2} \alpha\right)$ errors, our gluon action has $\mathcal{O}\left(a_{t}^{2}\right)$ errors from leaving out terms that give rise to unphysical branches. For anisotropies $a_{s} / a_{t} \geq 2$ the $\mathcal{O}\left(a_{t}^{2}\right)$ errors seem to be small. However, we have noticed that, as the coupling is varied, the ratio of string-tension to charmonium determinations of $a_{s}$ shows variations of about $5 \%$. This may be due to small violations of rotational invariance, and we are in the process of non-perturbatively tuning the gluon action to further reduce them [13].

For our preliminary investigation of quenched QCD on anisotropic lattices, we have chosen to study the D234 $\left(\frac{2}{3}\right)$ action (with the same TI prescription as for the glue) on 2:1 lattices for light quarks, and on 3:1 lattices for heavy masses. We use the SW action in various comparisons; both our as well as the results of [10] use TI. For our general simulation methodology we refer to [7].

For light quarks we performed simulations at three couplings, $\beta=2.0,2.15,2.3$, corresponding

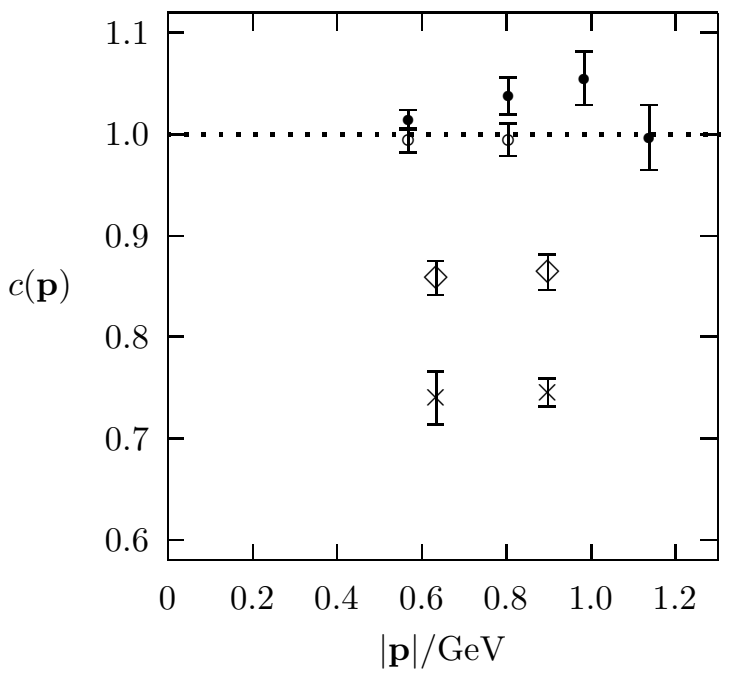

Figure 3. $\quad c(\mathbf{p})$ at various momenta for the D234 $\left(\frac{2}{3}\right)$ action on a $2: 1$ (pion $\bullet$, rho $\circ$ ) and the $\mathrm{SW}$ action on a 1:1 lattice (pion $\diamond$, rho $\times$ ). For both actions $m_{\rho} / m_{\pi} \approx 1.47$ and $a_{s} \approx 0.32 \mathrm{fm}$.

to lattice spacings $a_{s}=0.41,0.36,0.32 \mathrm{fm}$, if one uses charmonium to set the scale [13]. The spatial size of our lattices is about $2.4 \mathrm{fm}$ (smaller for large quark masses).

The first question is whether there are large $\mathcal{O}\left(a^{0}\right)$ effects violating space-time symmetry. As one can see in figure 3 , after TI such effects are very small. In addition, figures 3 and 5 show that $\mathrm{D} 234\left(\frac{2}{3}\right)$ is also more continuum-like at momenta up to $1 \mathrm{GeV}$, indicating that with TI the $\mathcal{O}\left(a^{2}\right)$ terms have roughly the right coefficients.

In figure 4 we see that the D234 $\left(\frac{2}{3}\right)$ rho mass, converted to physical units using the charmonium $\mathrm{S}-\mathrm{P}$ splitting, is closer to the continuum value on coarse lattices than for SW.

The value of $J[9]$ in table 1 is around $0.39(1)$ already on the coarsest lattices (as for isotropic D234 [7]); SW reaches this value only for much finer lattices [10]). The $m_{N} / m_{\rho}$ ratio appears rather high, especially at $\beta=2.3$, where it is larger than that of [7] as well as [10]. However, this might be due to chiral fitting uncertainties and problems with the glue.

Dramatic improvements are seen for the charmonium spectrum on anisotropic lattices. In fig- 


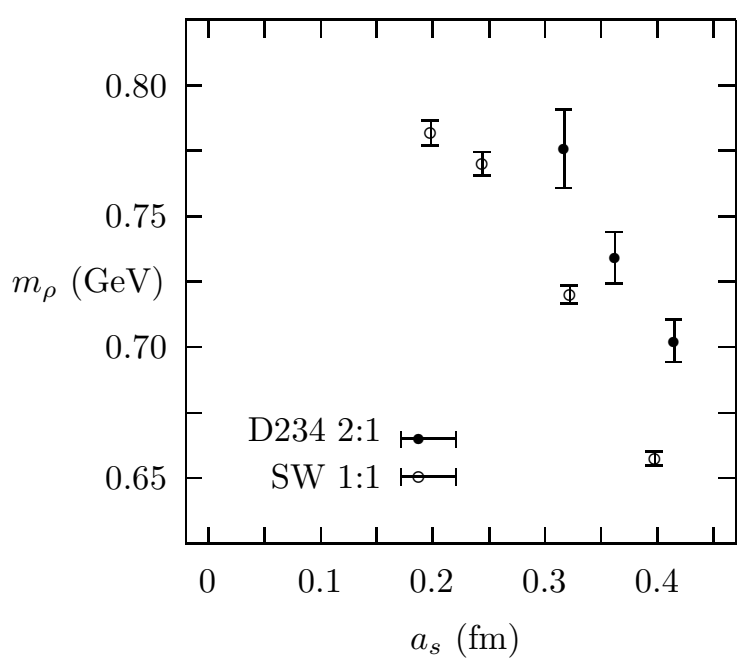

Figure 4. The rho mass for the 2:1 D234 $\left(\frac{2}{3}\right)$ and $1: 1 \mathrm{SW}[10]$ actions.

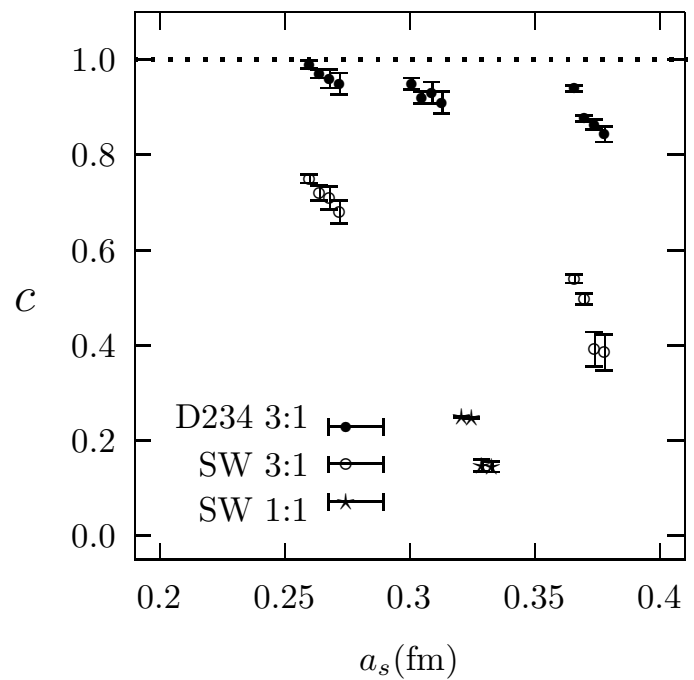

Figure 5. Effective velocity of light $c(\mathbf{p})$ for the D234 $\left(\frac{2}{3}\right)$ and SW actions on various lattices at the charm mass, $|\mathbf{p}| \approx 670 \mathrm{MeV}$. Each quadruplet represents, from left to right, the $\eta_{c}$ ("pion"), $J / \psi$ ("rho"), "nucleon" and "delta". For clarity we have slightly displaced the x-coordinates around the actual $a_{s}$.
Table 1

Comparison of 2:1 D234 $\left(\frac{2}{3}\right)$ and 1:1 SW [10]

\begin{tabular}{lllll}
\hline Action & $\beta$ & $a(\mathrm{fm})$ & $J$ & $m_{N} / m_{\rho}$ \\
\hline $\mathrm{D} 234$ & 2.0 & 0.41 & $0.380(5)$ & $1.37(2)$ \\
$\mathrm{D} 234$ & 2.15 & 0.36 & $0.396(9)$ & $1.32(5)$ \\
$\mathrm{D} 234$ & 2.3 & 0.32 & $0.375(6)(6)$ & $1.38(2)(3)$ \\
\hline SW & 6.8 & 0.40 & $0.345(4)$ & $1.457(15)$ \\
SW & 7.75 & 0.18 & $0.386(9)$ & $1.31(3)$ \\
\hline
\end{tabular}

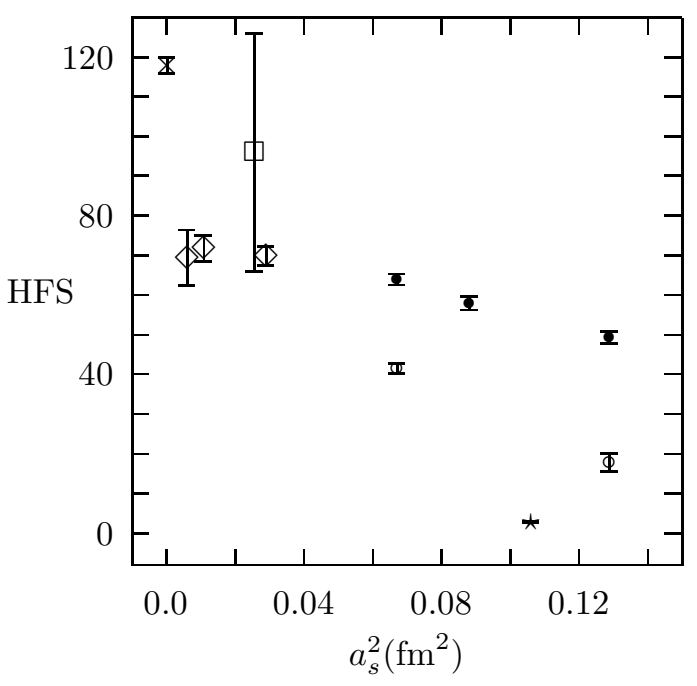

Figure 6. Quenched charmonium hyper-fine split-

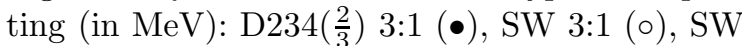
$1: 1(\star)$, FNAL $(\diamond)[16]$, NRQCD $(\square)$ [5], experiment $(x)$.

ures 5 and 6 we show results for D234 $\left(\frac{2}{3}\right)$ on $3: 1$ lattices and compare them with SW on 3:1 and 1:1 lattices. As expected, isotropic SW shows $O(1)$ scaling violations; SW on 3:1 is much better, and D234 $\left(\frac{2}{3}\right)$ on 3:1 is much better still.

Overall, anisotropic lattices are clearly well suited to coarse-lattice studies of heavy quarks and glueballs [12]. We have also seen some improvement in the rho mass for light quarks. However, our present results have systematic uncertainties in the glue and scale setting, so that it remains to be seen how big such improvements will ultimately be. For improved quark actions 
to be accurate on isotropic or anisotropic coarse lattices it might be necessary to eliminate $\mathcal{O}(a)$ quantum errors by non-perturbative tuning of the coefficients.

\section{Non-perturbative Tuning and the $r$-Test}

Our simulations so far rely on TI to estimate the coefficients in an action. We are now starting work on non-perturbative tuning. For an anisotropic action such a tuning might be necessary already at $\mathcal{O}\left(a^{0}\right)$, since the spatial and temporal first order derivatives might renormalize differently. As figure 3 shows, after TI this effect is small; the remainder could easily be tuned by introducing a "bare velocity of light" into the action and demanding $c(\mathbf{p})=1$ for small masses and momenta.

At $\mathcal{O}(a)$ one has to tune the clover term. Let us for the moment restrict ourselves to the isotropic case, where there are only two terms at $\mathcal{O}(a)$, which we write as $-\frac{a r}{2}\left(\sum_{\mu} \Delta_{\mu}+\frac{\omega}{2} \sigma \cdot F\right)$. The coefficient $r$, say, can be adjusted to any desired value by a field transformation. To eliminate quantum errors at $\mathcal{O}(a)$ one therefore has only to tune the (relative) clover coefficient $\omega$. A tuning method was presented in [14] for the SW action on Wilson glue, for which it was found that $\omega$ (at zero quark mass) is significantly larger than the TI estimate. Note that if this were also the case for the D234 action, it might solve the problem of its low rho mass.

An alternative method could be based on tuning $\omega$ so that the spectrum of the action is invariant under (small) changes of the Wilson $r$ parameter, cf. sect. 1. If $\omega$ were independent of $r$ (as it is classically) this will only be true for the correct non-perturbative $\omega$. The fact that $\omega$ presumably depends on $r$ at the quantum level, complicates the conversion of the $r$-test from a rough consistency check into a full tuning method. However, this is not a problem of principle, and we are presently exploring this idea.

On anisotropic lattices there is the additional complication, for both tuning and the $r$-test, that the temporal and spatial parts of the $\mathcal{O}(a)$ terms can suffer a relative renormalization. Assuming that TI makes this renormalization small, as we have seen for the $\mathcal{O}\left(a^{0}\right)$ terms, we can however also apply the $r$-test to anisotropic lattices. We have obtained preliminary results for the D234 actions of sect. 3. On a 2:1 lattice with $a_{s}=$ $0.36 \mathrm{fm}$ we measured the difference between the rho masses for $r=\frac{2}{3}$ and $r=1$ for (i) $\omega=0$, and (ii) the tree-level tadpole improved $\omega$. Tuning $m_{\rho} / m_{\pi}$ to be 1.3 in all cases, we find the change in the rho mass (in lattice units) to be 0.104(10) in the first case, and 0.012(4) in the second. This suggests that the correct $\omega$ is somewhat, but not dramatically larger than the TI estimate (even taking into account other results indicating that $\omega$ increases with decreasing mass).

\section{REFERENCES}

1. K. Symanzik, Nucl. Phys. B226 (1983) 187.

2. M. Lüscher and P. Weisz, Comm. Math. Phys. 97 (1985) 59; Phys. Lett. 158B (1985) 250.

3. B. Sheikholeslami and R. Wohlert, Nucl. Phys. B259 (1985) 572.

4. G.P. Lepage and P.B. Mackenzie, Phys. Rev. D48 (1993) 2250.

5. C. Davies et al Phys. Rev. D52 (1995) 6519; Phys. Lett. B345 (1995) 42.

6. M. Alford et al, Nucl. Phys. B42 (Proc. Suppl.) (1995) 787 and hep-lat/9507010.

7. M. Alford, T.R. Klassen, G.P. Lepage, Nucl. Phys. B (Proc. Suppl.) 47 (1996) 370.

8. H. Fiebig, F. Lewis and R. Woloshyn, these proceedings, hep-lat/9607058 and 9607059.

9. P. Lacock and C. Michael, Phys. Rev. D52 (1995) 5213.

10. S. Collins, R. Edwards, U. Heller and J. Sloan, these proceedings, hep-lat/9608021.

11. M. Alford, T.R. Klassen and G.P. Lepage, in preparation.

12. C. Morningstar, M. Peardon, these proceedings, hep-lat/9608050, hep-lat/9608019.

13. M. Alford, T.R. Klassen, G.P. Lepage, C. Morningstar, M. Peardon, and H. Trottier, to appear.

14. M. Lüscher et al, these proceedings, hep-lat/9605038, and hep-lat/9608049.

15. G. Heatlie et al, Nucl. Phys. B352 (1991) 266.

16. A.X. El-Khadra et al, in preparation. 